\title{
Investigation on the Genetic Signatures of Antibiotic Resistance in Multi-Drug-Resistant Klebsiella Pneumoniae Isolates From National Guard Hospital, Riyadh
}

\author{
Hassan N. Khdary ${ }^{1}$, Abdullah Almalki ${ }^{1}$, Mohamad H. Alkhdiri Jr. ${ }^{1}$, Saad Alhamoudi ${ }^{1}$, Abdullah Alfaleh ${ }^{1}$, \\ Majed F. Alghoribi ${ }^{2}$, Taher Uz Zaman ${ }^{2}$ \\ 1. Infectious Disease, King Saud Bin Abdulaziz University for Health Sciences College of Medicine, Riyadh, SAU 2. \\ Infectious Disease, King Abdullah International Medical Research Center, Riyadh, SAU
}

Corresponding author: Taher Uz Zaman, taher_zamans@yahoo.com

\section{Abstract \\ Introduction}

Despite a large number of antibiotics available to treat Klebsiella (K.) pneumoniae (KP), resistance against these antibiotics is ever-increasing and has now become a global threat to human life. The most frequently observed resistant genes in Klebsiella pneumoniae are CTX-M, OXA-48, IMP, and NDM; some are clonespecific while others form a reservoir for infection.

\section{Methods}

Matrix-assisted laser desorption ionization-time of flight (MALDI-TOF) was employed for the identification of the pathogens and automated VITEK-2 (bioMérieux, Marcy-l'Étoile, France) was used for minimum inhibitory concentration (MIC) determination, followed by polymerase chain reaction (PCR) amplification of target genes and Sanger sequencing of amplicons.

\section{Results}

Forty-three out of 50 isolates (86\%) were OXA gene-positive, and 49 out of 50 (98\%) isolates were CTX-M gene positive. Two phenotypes of OXA were identified in 33 samples sequenced, OXA-505 (70\%) and OXA$232(30 \%)$. Sixteen isolates (32\%) were positive for NDM-1. Twelve isolates were positive for both OXA and NDM. Multilocus sequence typing (MLST) on these isolates showed that they were distributed in 12 sequence types (STs). Thirty-six out of 50 were grouped in four clonal complexes. ST-14 was the predominant genotype.

\section{Conclusion}

Review began 10/14/2020 Review ended 10/23/2020 Published 11/01/2020

\section{(c) Copyright 2020}

Khdary et al. This is an open access article distributed under the terms of the Creative Commons Attribution License CC-BY 4.0., which permits unrestricted use, distribution, and reproduction in any medium, provided the original author and source are credited.
This study has revealed that CTX-M-15 is the most common extended-spectrum beta-lactamase (ESBL) present in almost all isolates. The study also shows the presence of OXA as the main carbapenemase gene, alone or in combination with other carbapenemases such as NDM-1. Multilocus sequence typing revealed the incidence of polyclonal KP pool with ST-14, ST-29, ST-307, and ST-15 being the predominant ones.

Categories: Infectious Disease

Keywords: klebsiella pneumoniae (kp), resistance genes, multi-locus sequence typing (mlst)

\section{Introduction}

Resistance against antibiotics is ever-increasing and has now become a global threat to human life [1-2]. Bacterial pathogens have developed resistance against many of these antibiotics, and some countries have reported strains that are resistant to all antibiotics available, i.e. pan-drug resistance [3]. Various mechanisms involving a battery of diversified genes are described for these resistances. Some of these genes are found on the bacterial chromosome, whereas others are found on episomal deoxyribonucleic acid (DNA) fragments called plasmids [4]. These plasmid-mediated genes can transfer resistance horizontally from one bacterium to another by inter-species or intra-species conjugations. The most frequently observed resistant genes in Klebsiella (K.) pneumoniae (KP) are TEM, SHV, CTX-M, OXA-48, IMP, VIM, NDM, and KPC. Some are clone-specific while others are transferred independently. Carbapenems are supposed to be the last line of drugs available against these pathogens. However, carbapenems have now also been reported to be ineffective due to the emergence of new resistant genes, such as KPC, OXA, NDM, VIM, and IMP, against this antibiotic [5]. Although the KPC gene is not much prevalent in Saudi Arabia, OXA-48 is widely reported in carbapenem-resistant Klebsiella pneumonia [6]. The New Delhi metallo-beta-lactamase 1 (NDM-1) gene is also being reported regularly from different parts of Saudi Arabia [7-8]. Few studies are available on the molecular basis of antibiotic resistance, virulence factors present, plasmid profiles carrying resistance genes, 
and the clonal diversity of these drug-resistant Klebsiella pneumoniae in Saudi Arabia. However, the true picture of antibiotic resistance in Klebsiella pneumoniae from the Kingdom in general and the National Guards Hospital, Riyadh, in particular still remains unclear. The aim of the present research is to understand the mechanisms of antibiotic resistance in multi-drug Carbapenem-resistant isolates of Klebsiella pneumoniae from the national guard hospital by investigating the genetic signatures of these resistant genes. We would also determine the genotypes of these strains that are predominant in this region to facilitate the control programs. All of this could help improve the infection control intervention and promote/support effective antibiotic stewardship programs in the National Guard Hospital, Saudi Arabia.

\section{Materials And Methods}

The study was conducted at the infectious diseases laboratory of King Abdullah International Medical Research Center (KAIMRC). Matrix-assisted laser desorption ionization-time of flight (MALDI-TOF) was employed for the identification of the pathogens and automated VITEK-2 (bioMérieux, Marcy-l'Étoile, France) was used for minimum inhibitory concentration (MIC) determination. The isolates, which showed elevated MIC for carbapenem and beta-lactam antibiotics and were designated as resistant by the microbiology department, National Guard Hospital, were the subject of this present study. Detection of resistance gene targets, such as CTX-M, OXA-48, and NDM, was done by polymerase chain reaction (PCR) using their specific primers. The sequencing of the gene products was done by the Sanger method to look for their variants (novel, if any). Multilocus sequence typing (MLST) was performed on these isolates using seven loci scheme proposed by Diancourt et al. [9] to determine their clonality and sequence type (ST).

\section{Data collection process}

Bacterial isolates were derived from patients on routine lab investigations. The sources of these isolates are given in Table 1. The identification was done by the MALDI-TOF technique in the microbiology lab. MICs were determined by VITEK-2. The KP isolates with elevated MIC were grown on blood agar medium. A single colony was selected and grown in a liquid broth medium. Fifty isolates of multi-drug carbapenem-resistance of Klebsiella pneumonia were investigated. DNA was extracted and PCR amplification of genes of antibiotic resistance (CTX-M, NDM, OXA) was done using the primers specific for these genes. These amplified products were visualized on an agarose gel by electrophoresis. Positive and negative controls were included. The confirmed positive PCR products were processed for sequencing by the Sanger Sequencing method. The DNA sequences were aligned and analyzed using Lasergene software. Mega 5 software was used for detecting single nucleotide polymorphisms (SNPs) and for phylogenetic analysis [10]. Each sequence was blasted against either the National Center for Biotechnology Information (NCBI) gene bank or a specific database for phenotype designation of these genes. The various phenotypes of the resistance genes of CTX-M, NDM, and OXA were determined and reported. Five researchers worked on the data collection process and the study took two years.

\section{Results}

\section{Demography}

The sources of these isolates are given in Table 1 .

\begin{tabular}{|c|c|c|c|c|c|}
\hline Isolate & Sequence type & Sampling Date & Specimen & Carbapenemase gene & Others \\
\hline RD-231 & 14 & 6/01/2015 & respiratory & OXA-232 + NDM & CTX-M \\
\hline RD-232 & 15 & 10/01/2015 & urine & NDM & CTX-M \\
\hline RD-233 & NA & 13/01/2015 & respiratory & OXA (phenotype NA) & CIX-M \\
\hline RD-234 & 15 & 18/01/2015 & wound & OXA-505 & CTX-M \\
\hline RD-235 & 22 & 23/01/2015 & wound & Negative & CTX-M \\
\hline RD-236 & 29 & 23/01/2015 & wound & OXA (phenotype NA) & CTX-M \\
\hline RD-237 & NA & 25/01/2015 & respiratory & OXA-505 & CTX-M \\
\hline RD-238 & 152 & 6/02/2015 & urine & OXA-505 + NDM & CTX-M \\
\hline RD-239 & 14 & 6/02/2015 & urine & OXA + NDM & CTX-M \\
\hline RD-240 & 29 & 8/02/2015 & respiratory & OXA-505 & CIX-M \\
\hline RD-241 & 15 & $12 / 02 / 2015$ & QC & OXA (phenotype NA) & CTX-M \\
\hline RD-242 & 29 & $12 / 02 / 2015$ & respiratory & OXA-505 & CTX-M \\
\hline RD-243 & 211 & 12/02/2015 & wound & OXA-505 & CTX-M \\
\hline
\end{tabular}




\section{Cureus}

\begin{tabular}{|c|c|c|c|c|c|}
\hline RD-244 & 29 & 21/02/2015 & respiratory & OXA-505 & СТХ-M \\
\hline RD-245 & NA & $10 / 03 / 2015$ & respiratory & OXA-505 & CTX-M \\
\hline RD-246 & 29 & $12 / 03 / 2015$ & blood & OXA (phenotype NA) & CTX-M \\
\hline RD-247 & 14 & $17 / 03 / 2015$ & respiratory & OXA-232 + NDM & CTX-M \\
\hline RD-248 & 29 & 27/03/2015 & wound & OXA-505 & CTX-M \\
\hline RD-249 & 14 & 2/04/2015 & respiratory & OXA-232 & CTX-M \\
\hline RD-250 & 15 & $15 / 04 / 2015$ & urine & Negative & CTX-M \\
\hline RD-251 & NA & 18/04/2015 & wound & NDM & Negative \\
\hline RD-252 & NA & $21 / 04 / 2015$ & urine & OXA-505 & CTX-M \\
\hline RD-253 & 716 & 22/04/2015 & blood & OXA-505 & CTX-M \\
\hline RD-254 & 15 & 27/04/2015 & blood & OXA-505 & CTX-M \\
\hline RD-255 & NA & 29/04/2015 & blood & NDM & CTX-M \\
\hline RD-256 & NA & $1 / 05 / 2015$ & wound & OXA (phenotype NA) & CTX-M \\
\hline RD-257 & NA & 6/05/2015 & blood & OXA (phenotype NA) & CTX-M \\
\hline RD-258 & NA & $5 / 05 / 2015$ & blood & OXA-505 & CTX-M \\
\hline RD-259 & 307 & 9/05/2015 & urine & OXA-505 & CTX-M \\
\hline RD-260 & 14 & $12 / 05 / 2015$ & wound & OXA-232 + NDM & CTX-M \\
\hline RD-261 & 14 & $15 / 05 / 2015$ & urine & OXA-232 + NDM & CTX-M \\
\hline RD-262 & 14 & 17/05/2015 & urine & OXA-232 & CTX-M \\
\hline RD-263 & NA & $13 / 05 / 2015$ & wound & OXA (phenotype NA) & CTX-M \\
\hline RD-264 & 11 & $22 / 05 / 2015$ & others & NDM & CTX-M \\
\hline RD-265 & 14 & $10 / 05 / 2015$ & blood & OXA-232 + NDM & CTX-M \\
\hline RD-266 & NA & $13 / 05 / 2015$ & blood & OXA (phenotype NA) & CTX-M \\
\hline RD-267 & 485 & $30 / 05 / 2015$ & urine & OXA-505 & CTX-M \\
\hline RD-268 & 14 & 3/06/2015 & urine & OXA-232 & CTX-M \\
\hline RD-269 & 14 & 10/06/2015 & blood & OXA + NDM & CTX-M \\
\hline RD-270 & 17 & $12 / 06 / 2015$ & Respiratory & OXA-505 & CTX-M \\
\hline RD-271 & 14 & $11 / 06 / 2015$ & blood & OXA-505 + NDM & CTX-M \\
\hline RD-272 & 1399 & $17 / 06 / 2015$ & wound & OXA-505 & CTX-M \\
\hline RD-273 & 307 & 18/06/2015 & blood & OXA-505 & CTX-M \\
\hline RD-274 & 307 & 19/06/2015 & blood & NDM & CTX-M \\
\hline RD-275 & 716 & 1/07/2015 & blood & OXA-505 & CTX-M \\
\hline RD-276 & NA & 1/07/2015 & urine & OXA-505 + NDM & CTX-M \\
\hline RD-277 & 14 & $2 / 07 / 2015$ & urine & OXA-232 + NDM & CTX-M \\
\hline RD-278 & 307 & 3/07/2015 & blood & OXA-505 & CTX-M \\
\hline RD-279 & 14 & 3/07/2015 & urine & OXA-505 & CTX-M \\
\hline RD-280 & 14 & $3 / 07 / 2015$ & urine & OXA-232 + NDM & CTX-M \\
\hline
\end{tabular}

TABLE 1: The demographic details and distribution of clonal types and antibiotic-resistant genes 
The clinical sources of the specimen for isolation of KP was as follows: respiratory $(n=10)$; surgical wound $(\mathrm{n}=10)$; $Q \mathrm{C}(\mathrm{n}=1)$; urine $(\mathrm{n}=14)$; blood $(\mathrm{n}=14)$; and Others $(\mathrm{n}=1)$.

\section{Multilocus sequence typing}

The multi-locus sequence typing (MLST) method using seven loci) showed 38 isolates being distributed in 12 STs. Twelve isolates could not be typed due to technical reasons. The distribution of STs was as follows: ST$14(\mathrm{n}=14)$, ST-15 $(\mathrm{n}=5)$, ST- $29(\mathrm{n}=6)$, ST-307 $(\mathrm{n}=4)$, ST-716 $(\mathrm{n}=2)$, ST-152 $(\mathrm{n}=1)$, ST-22 $(\mathrm{n}=1)$, ST-211 $(\mathrm{n}=1)$, ST$185(\mathrm{n}=1), \mathrm{ST}-17(\mathrm{n}=1)$, ST- $485(\mathrm{n}=1)$, and ST- $1399(\mathrm{n}=1)$. ST- 14 was the most common, with 14 isolates (28\%), followed by ST-29, ST-15, ST-307, and ST-716.

\begin{tabular}{|c|c|c|c|c|c|c|c|c|c|}
\hline MLST & $\mathbf{n}$ & CG & GapA & infB & mdh & pgi & phoE & гров & tonB \\
\hline 14 & 12 & \multirow{2}{*}{ C1 } & 1 & 6 & 1 & 1 & 1 & 1 & 1 \\
\hline 15 & 7 & & 1 & 1 & 1 & 1 & 1 & 1 & 1 \\
\hline 22 & 1 & \multirow{4}{*}{ C2 } & 2 & 3 & 1 & 1 & 1 & 4 & 4 \\
\hline 29 & 6 & & 2 & 3 & 2 & 2 & 1 & 4 & 4 \\
\hline 152 & 1 & & 2 & 3 & 2 & 1 & 1 & 4 & 56 \\
\hline 105 & 1 & & 2 & 3 & 2 & 1 & 1 & 4 & 18 \\
\hline 10002 & 1 & \multirow{3}{*}{ C3 } & 3 & 3 & 6 & 1 & 7 & 4 & 38 \\
\hline 10003 & 1 & & 3 & 3 & 1 & 1 & 9 & 4 & 43 \\
\hline 10006 & 1 & & 3 & 3 & 1 & 1 & 1 & 4 & 31 \\
\hline 10007 & 1 & \multirow{3}{*}{ C4 } & 2 & 5 & 1 & 1 & 7 & 1 & 15 \\
\hline 2648 & 2 & & 2 & 5 & 1 & 1 & 7 & 1 & 6 \\
\hline 4698 & 2 & & 2 & 5 & 1 & 2 & 7 & 1 & 10 \\
\hline
\end{tabular}

\section{TABLE 2: MLST complexes}

MLST: multilocus sequence typing

\section{Resistance genes profile}

All isolates were resistant to more than three types of antibiotics for MIC. The antibiogram profile of these isolates is given in Table 1 .

All isolates were resistant to more than three types of antibiotics. CTX-M was the most prevalent, with 49 out of 50 (98\%) positive samples, and was carried by isolates of all STs. All the representative 28 samples sequenced were CTX-15. The OXA gene was the most common carbapenemase gene. Sequence analysis of the $~ 740$-base pair PCR product revealed an OXA gene allele sensu stricto in all the positive isolates. Fortythree out of 50 (86\%) of the samples were positive for OXA. Twenty-three out of 33 sequenced samples (70\%) were OXA-505 while 10 (30\%) were OXA-232. The phenotypes of the remaining OXA positive isolates could not be determined because of some technical reasons. Twenty-eight out of 50 of the samples (56\%) were positive for NDM. The seven amplicons that were subjected to sequencing turned out to be NDM-1. No other allelic form of the NDM gene was detected in any isolate. The co-existence of two carbapenemase genes (NDM-1 + OXA) was seen in $12(24 \%)$ isolates. Two isolates, one each from ST-22 and ST-15, were negative for both the OXA and NDM-1 genes. The CTX-M-15 gene allele was the major extended-spectrum $\beta$-lactamase (ESBL), with gene positivity in 49 isolates (98\%). Details are given in Table 3. ST-14 and ST-15 were the predominant clones of the study population. The phylogenetic relationship of these isolates is shown in Figure 1. 


\begin{tabular}{|c|c|c|c|c|c|c|c|c|c|c|c|c|c|c|c|}
\hline 231 & 6/1/2015 & resp & $\mathrm{N}$ & $32 \mathrm{R}$ & $128 \mathrm{R}$ & $64 \mathrm{R}$ & $1 \mathrm{~S}$ & $64 \mathrm{R}$ & $64 \mathrm{R}$ & $16 R$ & $16 \mathrm{R}$ & $4 \mathrm{R}$ & $256 \mathrm{R}$ & $320 \mathrm{R}$ & $8 \mathrm{R}$ \\
\hline 232 & 10/1/2015 & urine & $\mathrm{N}$ & $32 \mathrm{R}$ & $128 \mathrm{R}$ & $64 \mathrm{R}$ & $16 \mathrm{R}$ & $64 \mathrm{R}$ & $64 \mathrm{R}$ & $16 \mathrm{R}$ & $16 \mathrm{R}$ & $4 \mathrm{R}$ & $128 \mathrm{R}$ & $320 \mathrm{R}$ & $1 \mathrm{~S}$ \\
\hline 233 & 13/1/2015 & resp & $\mathrm{N}$ & $32 \mathrm{R}$ & $128 \mathrm{R}$ & $64 \mathrm{R}$ & $1 \mathrm{~S}$ & $64 \mathrm{R}$ & 2I & $8 \mathrm{R}$ & 21 & $1 \mathrm{~S}$ & $128 \mathrm{R}$ & $20 \mathrm{~S}$ & 41 \\
\hline 234 & 18/1/2015 & wound & NA & $32 R$ & $128 \mathrm{R}$ & $2 S$ & $1 \mathrm{~S}$ & NA & $64 \mathrm{R}$ & $16 \mathrm{R}$ & $16 \mathrm{R}$ & $4 \mathrm{R}$ & NA & $20 \mathrm{~S}$ & $4 I$ \\
\hline 235 & 23/01/2015 & wound & $\mathrm{N}$ & $32 \mathrm{R}$ & $128 \mathrm{R}$ & 321 & $16 \mathrm{R}$ & $64 \mathrm{R}$ & $64 \mathrm{R}$ & $8 \mathrm{R}$ & $8 \mathrm{R}$ & $4 \mathrm{R}$ & $128 \mathrm{R}$ & $320 \mathrm{R}$ & $8 \mathrm{R}$ \\
\hline 236 & $23 / 01 / 2015$ & wound & NA & $32 \mathrm{R}$ & $128 \mathrm{R}$ & $64 \mathrm{R}$ & $16 \mathrm{R}$ & NA & $64 \mathrm{R}$ & $16 \mathrm{R}$ & $16 \mathrm{R}$ & $4 \mathrm{R}$ & NA & $320 \mathrm{R}$ & $4 I$ \\
\hline 237 & $25 / 01 / 2015$ & resp & $\mathrm{N}$ & $32 \mathrm{R}$ & $128 \mathrm{R}$ & $2 S$ & $1 \mathrm{~S}$ & $64 \mathrm{R}$ & 81 & 21 & 11 & $0.25 \mathrm{~S}$ & $64 I$ & $20 \mathrm{~S}$ & $1 \mathrm{~S}$ \\
\hline 238 & $26 / 02 / 2015$ & urine & NA & $32 \mathrm{R}$ & $128 \mathrm{R}$ & $64 \mathrm{R}$ & $1 \mathrm{~S}$ & NA & $64 \mathrm{R}$ & $16 \mathrm{R}$ & $16 \mathrm{R}$ & $4 \mathrm{R}$ & NA & $320 \mathrm{R}$ & $8 \mathrm{R}$ \\
\hline 239 & $26 / 02 / 2015$ & urine & $\mathrm{N}$ & $32 \mathrm{R}$ & $128 \mathrm{R}$ & $64 \mathrm{R}$ & $16 \mathrm{R}$ & $64 \mathrm{R}$ & $64 \mathrm{R}$ & $16 \mathrm{R}$ & $16 \mathrm{R}$ & $4 \mathrm{R}$ & $256 \mathrm{R}$ & $320 \mathrm{R}$ & $8 \mathrm{R}$ \\
\hline 240 & $8 / 2 / 2015$ & resp & NA & $32 R$ & $128 \mathrm{R}$ & $64 R$ & $1 \mathrm{~S}$ & NA & $16 \mathrm{I}$ & $16 \mathrm{R}$ & 11 & $4 \mathrm{R}$ & NA & $320 R$ & $4 \mathrm{I}$ \\
\hline 241 & 12/2/2015 & qc & $\mathrm{P}$ & $32 \mathrm{R}$ & $128 \mathrm{R}$ & $2 \mathrm{~S}$ & $16 \mathrm{R}$ & $64 \mathrm{R}$ & 161 & 21 & 11 & $4 \mathrm{R}$ & $128 \mathrm{R}$ & $320 \mathrm{R}$ & $8 \mathrm{R}$ \\
\hline 242 & 12/2/2015 & resp & $\mathrm{N}$ & $32 \mathrm{R}$ & $128 \mathrm{R}$ & $64 \mathrm{R}$ & $16 \mathrm{R}$ & $64 \mathrm{R}$ & 81 & $16 \mathrm{R}$ & 11 & $4 \mathrm{R}$ & $128 \mathrm{R}$ & $320 \mathrm{R}$ & 41 \\
\hline 243 & 12/2/2015 & wound & $\mathrm{P}$ & $32 \mathrm{R}$ & $128 \mathrm{R}$ & $64 \mathrm{R}$ & $1 \mathrm{~S}$ & $64 \mathrm{R}$ & 2I & $16 \mathrm{R}$ & $16 \mathrm{R}$ & $0.25 \mathrm{~S}$ & $64 I$ & $60 \mathrm{~S}$ & $1 \mathrm{~S}$ \\
\hline 244 & 21/2/2015 & resp & NA & $32 \mathrm{R}$ & $128 \mathrm{R}$ & $64 \mathrm{R}$ & $16 \mathrm{R}$ & NA & $64 \mathrm{R}$ & $16 \mathrm{R}$ & 11 & $4 \mathrm{R}$ & NA & $320 \mathrm{R}$ & $4 I$ \\
\hline 245 & 10/3/2015 & resp & $\mathrm{N}$ & $32 \mathrm{R}$ & $128 \mathrm{R}$ & $2 S$ & $1 \mathrm{~S}$ & $64 \mathrm{R}$ & $4 I$ & $16 \mathrm{R}$ & 11 & $0.25 \mathrm{~S}$ & 641 & $20 \mathrm{~S}$ & $0.5 \mathrm{~S}$ \\
\hline 246 & 2/4/2015 & blood & $\mathrm{N}$ & $32 \mathrm{R}$ & $128 \mathrm{R}$ & $64 \mathrm{R}$ & $1 \mathrm{~S}$ & $64 \mathrm{R}$ & $64 \mathrm{R}$ & $16 \mathrm{R}$ & 11 & $4 \mathrm{R}$ & $64 I$ & $320 \mathrm{R}$ & 41 \\
\hline 247 & 17/3/2015 & resp & $\mathrm{N}$ & $32 \mathrm{R}$ & $128 \mathrm{R}$ & $64 \mathrm{R}$ & $16 \mathrm{R}$ & $64 \mathrm{R}$ & $64 \mathrm{R}$ & $16 \mathrm{R}$ & $16 \mathrm{R}$ & $4 \mathrm{R}$ & $256 \mathrm{R}$ & $320 \mathrm{R}$ & $8 \mathrm{R}$ \\
\hline 248 & 27/3/2015 & wound & $\mathrm{N}$ & $32 \mathrm{R}$ & $128 \mathrm{R}$ & $64 \mathrm{R}$ & $16 \mathrm{R}$ & $64 \mathrm{R}$ & $64 \mathrm{R}$ & $16 \mathrm{R}$ & $16 \mathrm{R}$ & $4 \mathrm{R}$ & $256 \mathrm{R}$ & $320 \mathrm{R}$ & $8 \mathrm{R}$ \\
\hline 249 & 12/3/2015 & resp & $\mathrm{N}$ & $32 \mathrm{R}$ & $128 \mathrm{R}$ & $64 \mathrm{R}$ & 41 & $64 \mathrm{R}$ & $64 \mathrm{R}$ & $16 \mathrm{R}$ & $16 \mathrm{R}$ & $4 \mathrm{R}$ & $512 R$ & $320 \mathrm{R}$ & $8 \mathrm{R}$ \\
\hline 250 & $15 / 4 / 2015$ & urine & $\mathrm{N}$ & $32 \mathrm{R}$ & $128 \mathrm{R}$ & $16 \mathrm{~S}$ & $1 \mathrm{~S}$ & $64 \mathrm{R}$ & $64 \mathrm{R}$ & $16 R$ & $16 \mathrm{R}$ & $4 \mathrm{R}$ & $256 \mathrm{R}$ & $40 \mathrm{~S}$ & $8 \mathrm{R}$ \\
\hline 251 & 18/4/2015 & wound & $\mathrm{N}$ & $32 \mathrm{R}$ & $128 \mathrm{R}$ & $2 S$ & $1 \mathrm{~S}$ & $64 \mathrm{R}$ & 81 & $8 \mathrm{R}$ & $8 \mathrm{R}$ & $4 \mathrm{R}$ & $256 \mathrm{R}$ & $20 \mathrm{~S}$ & $8 \mathrm{R}$ \\
\hline 252 & 21/4/2015 & urine & $\mathrm{N}$ & $32 \mathrm{R}$ & $128 \mathrm{R}$ & $2 S$ & $1 \mathrm{~S}$ & $64 \mathrm{R}$ & 2 I & 21 & 11 & $0.25 \mathrm{~S}$ & $64 \mid$ & $20 \mathrm{~S}$ & $0.5 \mathrm{~S}$ \\
\hline 253 & 22/4/2015 & blood & NA & $32 \mathrm{R}$ & $128 \mathrm{R}$ & $2 S$ & $16 \mathrm{R}$ & NA & 21 & 21 & 4R & $2 \mathrm{R}$ & NA & $320 \mathrm{R}$ & $2 S$ \\
\hline 254 & 27/4/2015 & blood & $\mathrm{N}$ & $32 \mathrm{R}$ & $128 \mathrm{R}$ & $2 \mathrm{~S}$ & $16 \mathrm{R}$ & $64 \mathrm{R}$ & $64 \mathrm{R}$ & $8 \mathrm{R}$ & $4 \mathrm{R}$ & $4 \mathrm{R}$ & $256 \mathrm{R}$ & $320 \mathrm{R}$ & $8 \mathrm{R}$ \\
\hline 255 & 29/4/2015 & blood & $\mathrm{P}$ & $32 \mathrm{R}$ & $128 \mathrm{R}$ & $64 \mathrm{R}$ & $16 \mathrm{R}$ & $64 \mathrm{R}$ & 81 & $16 \mathrm{R}$ & 11 & $1 \mathrm{~S}$ & $128 \mathrm{R}$ & $320 \mathrm{R}$ & $0.5 \mathrm{~S}$ \\
\hline 256 & 1/5/2015 & wound & $\mathrm{N}$ & $32 \mathrm{R}$ & $128 \mathrm{R}$ & $16 \mathrm{~S}$ & $2 \mathrm{~S}$ & $64 \mathrm{R}$ & $64 \mathrm{R}$ & $16 \mathrm{R}$ & $16 \mathrm{R}$ & $0.5 \mathrm{~S}$ & $64 I$ & $20 \mathrm{~S}$ & $1 \mathrm{~S}$ \\
\hline 257 & 6/5/2015 & blood & $\mathrm{N}$ & $32 \mathrm{R}$ & $128 \mathrm{R}$ & $64 \mathrm{R}$ & $16 \mathrm{R}$ & $64 \mathrm{R}$ & $64 \mathrm{R}$ & $4 \mathrm{R}$ & $16 \mathrm{R}$ & $4 \mathrm{R}$ & $512 R$ & $20 \mathrm{~S}$ & $2 S$ \\
\hline 258 & 5/5/2015 & blood & $\mathrm{N}$ & $32 \mathrm{R}$ & $128 \mathrm{R}$ & $64 \mathrm{R}$ & $1 \mathrm{~S}$ & $64 \mathrm{R}$ & $64 \mathrm{R}$ & $16 \mathrm{R}$ & $16 \mathrm{R}$ & $4 \mathrm{R}$ & $256 \mathrm{R}$ & $320 \mathrm{R}$ & 41 \\
\hline 259 & 9/5/2015 & urine & $P$ & $32 R$ & $128 \mathrm{R}$ & $64 \mathrm{R}$ & $16 R$ & $64 R$ & 161 & $4 R$ & 11 & $4 \mathrm{R}$ & $256 \mathrm{R}$ & $320 R$ & $8 \mathrm{R}$ \\
\hline 260 & 12/5/2015 & wound & NA & $32 \mathrm{R}$ & $128 \mathrm{R}$ & $64 \mathrm{R}$ & $16 \mathrm{R}$ & $64 \mathrm{R}$ & $64 \mathrm{R}$ & $16 \mathrm{R}$ & $16 \mathrm{R}$ & $4 \mathrm{R}$ & $256 \mathrm{R}$ & $320 \mathrm{R}$ & $8 \mathrm{R}$ \\
\hline 261 & $15 / 5 / 2015$ & urine & $\mathrm{N}$ & $32 R$ & $128 \mathrm{R}$ & $64 \mathrm{R}$ & $4 I$ & $64 \mathrm{R}$ & $64 \mathrm{R}$ & $16 R$ & $16 R$ & $4 \mathrm{R}$ & $256 \mathrm{R}$ & $320 \mathrm{R}$ & $8 \mathrm{R}$ \\
\hline 262 & $17 / 5 / 2015$ & urine & $\mathrm{N}$ & $32 R$ & $128 \mathrm{R}$ & 321 & $1 \mathrm{~S}$ & $64 \mathrm{R}$ & $64 \mathrm{R}$ & 21 & $16 \mathrm{R}$ & $4 \mathrm{R}$ & $256 \mathrm{R}$ & $320 \mathrm{R}$ & $8 \mathrm{R}$ \\
\hline 263 & $13 / 5 / 2015$ & wound & $P$ & $32 R$ & $128 \mathrm{R}$ & $64 \mathrm{R}$ & $1 \mathrm{~S}$ & $64 \mathrm{R}$ & 21 & 21 & $0.5 \mathrm{I}$ & $0.25 \mathrm{~S}$ & $64 \mid$ & $20 \mathrm{~S}$ & $0.5 \mathrm{~S}$ \\
\hline 264 & 22/5/2015 & other & NA & $32 \mathrm{R}$ & $128 \mathrm{R}$ & $2 S$ & $1 \mathrm{~S}$ & NA & $64 \mathrm{R}$ & $16 \mathrm{R}$ & $16 \mathrm{R}$ & $4 \mathrm{R}$ & NA & $40 \mathrm{~S}$ & $4 I$ \\
\hline 265 & $10 / 5 / 2015$ & blood & $\mathrm{N}$ & $32 R$ & $128 \mathrm{R}$ & $64 \mathrm{R}$ & 81 & $64 \mathrm{R}$ & $64 \mathrm{R}$ & $16 R$ & $16 R$ & $4 \mathrm{R}$ & $256 \mathrm{R}$ & $320 \mathrm{R}$ & $8 \mathrm{R}$ \\
\hline 266 & 13/5/2015 & blood & $\mathrm{P}$ & $32 R$ & $128 \mathrm{R}$ & $64 \mathrm{R}$ & $1 \mathrm{~S}$ & $64 \mathrm{R}$ & 2I & 21 & 11 & $0.25 \mathrm{~S}$ & $64 I$ & $20 \mathrm{~S}$ & $0.5 \mathrm{~S}$ \\
\hline 267 & $30 / 5 / 2015$ & urine & $P$ & $32 R$ & $128 \mathrm{R}$ & $16 \mathrm{~S}$ & $1 \mathrm{~S}$ & $64 R$ & 81 & 2I & 11 & $4 \mathrm{R}$ & $128 \mathrm{R}$ & $40 \mathrm{~S}$ & $2 \mathrm{~S}$ \\
\hline 268 & $3 / 6 / 2015$ & urine & $\mathrm{N}$ & $32 R$ & $128 \mathrm{R}$ & $64 \mathrm{R}$ & $16 \mathrm{R}$ & $64 \mathrm{R}$ & $64 \mathrm{R}$ & $16 \mathrm{R}$ & $16 \mathrm{R}$ & $4 \mathrm{R}$ & $256 \mathrm{R}$ & $320 \mathrm{R}$ & $8 \mathrm{R}$ \\
\hline 269 & 10/6/2015 & blood & $\mathrm{N}$ & $32 R$ & $128 R$ & $64 R$ & $16 \mathrm{R}$ & $64 \mathrm{R}$ & $64 \mathrm{R}$ & $16 \mathrm{R}$ & $16 \mathrm{R}$ & $4 \mathrm{R}$ & $256 \mathrm{R}$ & $320 \mathrm{R}$ & $8 \mathrm{R}$ \\
\hline
\end{tabular}




\section{Cureus}

\begin{tabular}{|c|c|c|c|c|c|c|c|c|c|c|c|c|c|c|c|}
\hline 270 & $12 / 6 / 2015$ & resp & $\mathbf{P}$ & $32 R$ & $128 \mathrm{R}$ & $2 S$ & $1 \mathrm{~S}$ & $64 \mathrm{R}$ & 2I & $16 \mathrm{R}$ & 11 & $0.25 \mathrm{~S}$ & $32 \mathrm{~S}$ & $320 \mathrm{R}$ & 1S \\
\hline 271 & 11/6/2015 & blood & NA & $32 \mathrm{R}$ & $128 \mathrm{R}$ & $2 S$ & $1 \mathrm{~S}$ & NA & $64 \mathrm{R}$ & $16 \mathrm{R}$ & $16 \mathrm{R}$ & $4 \mathrm{R}$ & NA & $20 \mathrm{~S}$ & $8 \mathrm{R}$ \\
\hline 272 & 17/6/2015 & wound & NA & $32 R$ & $128 \mathrm{R}$ & $16 \mathrm{~S}$ & $16 \mathrm{R}$ & NA & $64 \mathrm{R}$ & 2I & 11 & $4 \mathrm{R}$ & NA & $320 \mathrm{R}$ & $4 I$ \\
\hline 273 & 18/6/2015 & blood & $\mathrm{N}$ & $32 \mathrm{R}$ & $128 \mathrm{R}$ & $2 S$ & $1 \mathrm{~S}$ & $64 \mathrm{R}$ & 64R & $16 \mathrm{R}$ & $16 \mathrm{R}$ & $4 \mathrm{R}$ & $256 \mathrm{R}$ & $20 \mathrm{~S}$ & 41 \\
\hline 274 & 19/6/2015 & blood & $\mathrm{N}$ & $32 R$ & $128 \mathrm{R}$ & $16 \mathrm{~S}$ & $16 R$ & $64 R$ & $64 R$ & $16 R$ & $16 \mathrm{R}$ & $4 \mathrm{R}$ & $64 \mid$ & $320 R$ & $2 S$ \\
\hline 275 & 1/7/2015 & blood & $\mathrm{P}$ & $32 R$ & $128 \mathrm{R}$ & $64 \mathrm{R}$ & $16 \mathrm{R}$ & $64 \mathrm{R}$ & 81 & 21 & 11 & $2 \mathrm{R}$ & $64 I$ & $320 \mathrm{R}$ & $2 S$ \\
\hline 276 & 1/7/2015 & urine & $\mathrm{N}$ & $32 R$ & $128 \mathrm{R}$ & $64 \mathrm{R}$ & $1 \mathrm{~S}$ & $64 \mathrm{R}$ & 161 & 21 & 11 & $4 \mathrm{R}$ & $128 \mathrm{R}$ & $40 \mathrm{~S}$ & $8 R$ \\
\hline 277 & 3/7/2015 & urine & $\mathrm{N}$ & $32 \mathrm{R}$ & $128 \mathrm{R}$ & $64 \mathrm{R}$ & $16 \mathrm{R}$ & $64 \mathrm{R}$ & 64R & $16 \mathrm{R}$ & 16R & $4 \mathrm{R}$ & $512 R$ & $320 \mathrm{R}$ & $8 \mathrm{R}$ \\
\hline 278 & 3/7/2015 & blood & $\mathrm{P}$ & $32 \mathrm{R}$ & $128 \mathrm{R}$ & $64 \mathrm{R}$ & $1 \mathrm{~S}$ & $64 R$ & $4 I$ & 21 & 11 & $4 \mathrm{R}$ & $128 \mathrm{R}$ & $320 R$ & $8 R$ \\
\hline 279 & 3/7/2015 & urine & $\mathrm{N}$ & $32 \mathrm{R}$ & $128 \mathrm{R}$ & $2 S$ & $1 \mathrm{~S}$ & $64 \mathrm{R}$ & 161 & 2I & 11 & 4R & $128 \mathrm{R}$ & $20 \mathrm{~S}$ & $8 \mathrm{R}$ \\
\hline 280 & $5 / 7 / 2015$ & blood & $\mathrm{N}$ & $32 R$ & $128 \mathrm{R}$ & $64 R$ & $16 R$ & $64 R$ & $64 R$ & $16 R$ & $16 \mathrm{R}$ & $4 R$ & $256 \mathrm{R}$ & $320 R$ & $8 R$ \\
\hline
\end{tabular}

TABLE 3: Resistance genes profile

C1

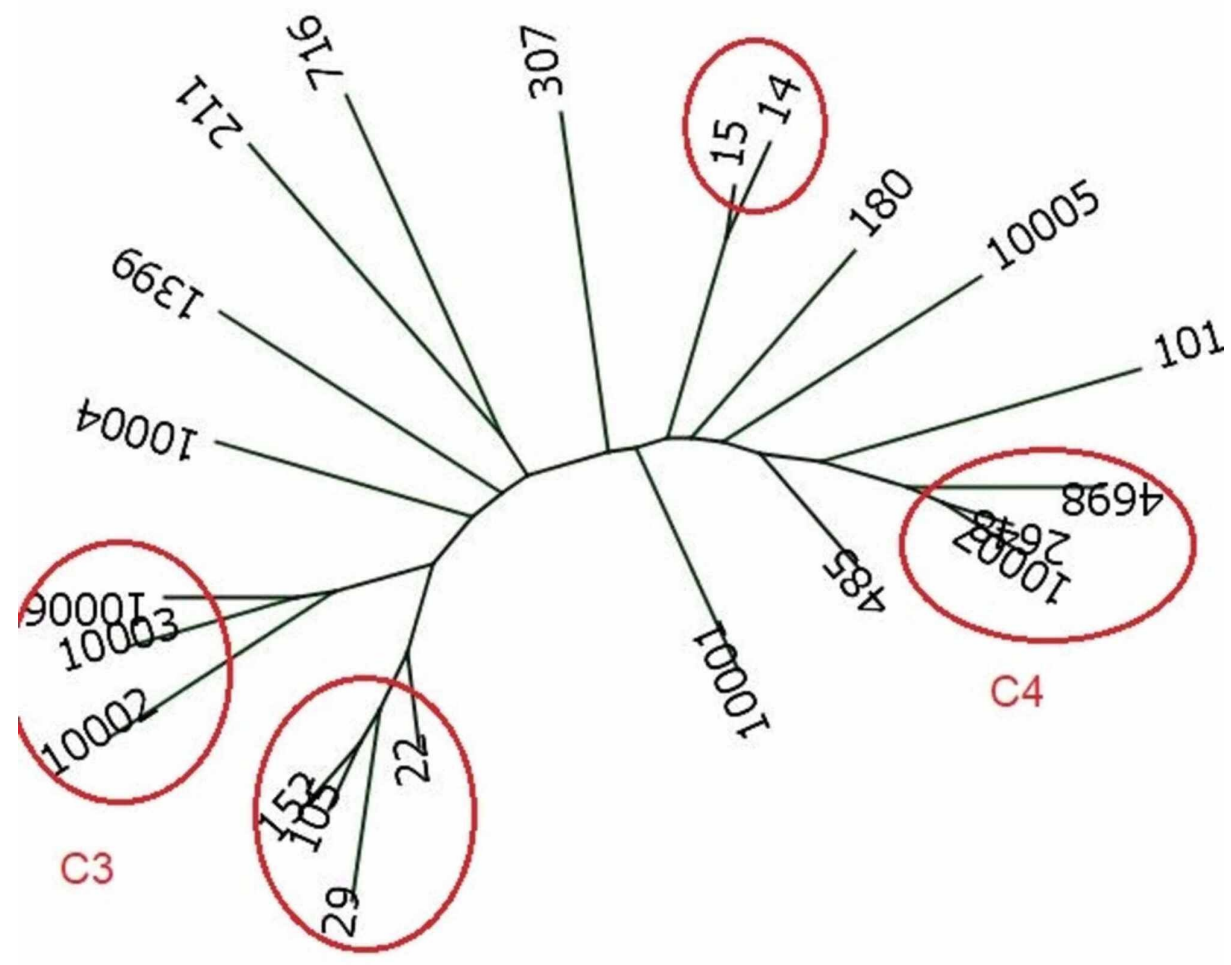

C2

FIGURE 1: Phylogenic tree

\section{Discussion}

Multi-drug resistance among KP has become a world phenomenon, nullifying the benefits of high-profile clinical skills such as open-heart and transplantation surgeries [11]. Several reports have projected Saudi Arabia as a growing pool for such multi-drug resistant pathogens because of several reasons [11-13]. In addition to common ESBLs, the incidence of CTX-M and other carbapenems are on the increase [14]. Here, we report that all our isolates were positive for CTX-M-15, underlying the multi-drug resistant profiles of these isolates. CTX-M-15 has previously been reported to be dominant among KP isolates from Saudi Arabia and has also been reported among outbreak isolates elsewhere, where $55 \%$ of Klebsiella pneumoniae isolates 
showed the phenotypic characterization of high ESBL [14-15]. The Klebsiella pneumoniae-producing ESBL were positive for the SHV, TEM, and CTX-M $\beta$-lactamase genes; the CTX-M genes that were more prevalent were CTX-M-1 and CTX-M-9-like genes [14-15]. CTX-M-15 was present in isolates from all STs, indicating its epidemicity. The OXA-48 gene has previously been reported in MDR-KP isolates from National Guard Health Affairs (NGHA), Riyadh. Our present study also showed the presence of OXA-505 (a member of the OXA-48 family) in as many as $70 \%$ of studied isolates. OXA-232 is reported for the first time in our KP isolates $[6,8,11]$. These findings need further investigations in a larger number of samples. The phylogenetic relationship between those two phenotypes shows clear isolation between these two groups (Figure 1).

The incidence of NDM in as much as $32 \%$ of isolates is in agreement with the previous reports from Saudi Arabia $[8,11,16]$. The coexistence of OXA and NDM genes also has been previously reported and underpins the elevated MIC levels of Carbapenem for these isolates. None of the resistance genes is clone specific and evenly distributed among all sequence types of KP (Table 1).

A polyclonal KP population was observed with 12 different STs. ST-14, ST-29, and ST-15 were the predominant clones of our study population. Most of these STs are frequently reported from different parts of the globe $[17,18]$. Thirty-seven isolates formed four clonal complexes based on similarities in four or more loci out of the seven loci of the MLST scheme. ST-14 and ST-15 are single locus variants (SLV) of each other differing in one locus. ST-14, ST-15, and ST-29 have been previously reported from this region. ST-14 is now being considered as an endemic clone for colistin resistance [19]. The large numbers of certain sequence types might be due to their clonal expansion [20]. Genetic diversity and population structure have inferences, which have been drawn by the MLST tool. This study has shown several genetically related antibiotic-resistant KP STs grouped into at least four clonal complexes based on mono-, di-, and tri-locus variations and are designated as C1-C4 for our study (Table 2). In members of complex C1, there is a single locus variation in infB among their isolates, whereas in complex $\mathrm{C} 2$ and $\mathrm{C} 3$, there is a tri-locus variation involving the mdh, pgi, phoE, and tonB genes. Finally, complex $\mathrm{C} 4$ has a di-locus variation in pgi mad tonB genes. As we see, three complexes have locus variations in tonB, which are $\mathrm{C} 2, \mathrm{C} 3$, and C4.

The main limitation of this study is that these results might not give the true picture of antibiotic resistance in the whole country. A multi-centric study involving a larger number of isolates is needed to reach a meaningful conclusion and a true picture of antibiotic resistance in the Kingdom. Investigations on the plasmids carrying the resistance genes would also shed light on the horizontal transfer of these resistant genes. However, our study forms a base for such investigations.

\section{Conclusions}

The study has revealed the presence of CTX-M-15 in almost all isolates. The study also shows the presence of carbapenemase genes, such as OXA and NDM, singularly or in combination. Newer OXA phenotypes, such as OXA-232 and OXA-505, were detected. Multilocus sequence typing revealed the incidence of a polyclonal KP pool, ST14 and ST15 being the predominant ones. Further studies should be focused on these lines to generate comprehensive data to form a solid database of an antibiotic-resistant gene profile among the bacterial pathogens in Saudi Arabia.

\section{Additional Information \\ Disclosures}

Human subjects: Consent was obtained by all participants in this study. Institutional Review Board of King Abdulaziz Medical City issued approval H-01-R-005. Animal subjects: All authors have confirmed that this study did not involve animal subjects or tissue. Conflicts of interest: In compliance with the ICMJE uniform disclosure form, all authors declare the following: Payment/services info: All authors have declared that no financial support was received from any organization for the submitted work. Financial relationships: All authors have declared that they have no financial relationships at present or within the previous three years with any organizations that might have an interest in the submitted work. Other relationships: All authors have declared that there are no other relationships or activities that could appear to have influenced the submitted work.

\section{Acknowledgements}

The authors acknowledge the technical help from Maha Albladi and Shahad Haddad (KAIMRC). Special thanks to Dr. Amir Umair, department of Medical Education, College of Medicine, KSAU-HS, Riyadh, for the coordination with the medical students involved in this project.

\section{References}

1. Munoz-Price LS, Poirel L, Bonomo RA, et al.: Clinical epidemiology of the global expansion of Klebsiella pneumoniae carbapenemases. Lancet Infect Dis. 2013, 13:785-796. 10.1016/S1473-3099(13)70190-7

2. Sonnevend A, Ghazawi AA, Hashmey R, et al.: Characterization of carbapenem-resistant Enterobacteriaceae with high rate of autochthonous transmission in the Arabian peninsula. PLoS One. 2015, 10:0131372. 10.1371/journal.pone.0131372 
3. Woodford N, Turton JF, Livermore DM: Multiresistant gram-negative bacteria: the role of high-risk clones in the dissemination of antibiotic resistance. FEMS Microbiol Rev. 2011, 35:736-755. 10.1111/j.15746976.2011.00268.x

4. Lee MY, Ko KS, Kang CI, Ryeon D, Kyong C, Peck R, Songac J-H: High prevalence of CTX-M-15-producing Klebsiella pneumoniae isolates in Asian countries: diverse clones and clonal dissemination. Int J Antimicrob Agents. 2011, 38:160-163. 10.1016/j.ijantimicag.2011.03.020

5. Poirel L, Potron A, Nordmann P: OXA-48-like carbapenemases: the phantom menace. J Antimicrob Chemother. 2012, 67:1597-1606. 10.1093/jac/dks121

6. Uz Zaman T, Aldrees M, Al Johani SM, Alrodayyan M, Aldughashem FA, Balkhy HH: Multi-drug carbapenemresistant Klebsiella pneumoniae infection carrying the OXA-48 gene and showing variations in outer membrane protein 36 causing an outbreak in a tertiary care hospital in Riyadh, Saudi Arabia. Int J Infect Dis. 2014, 28:186-192. 10.1016/j.ijid.2014.05.021

7. Zowawi HM, Sartor AL, Balkhy HH, et al.: Molecular characterization of carbapenemase-producing Escherichia coli and Klebsiella pneumoniae in the countries of the Gulf Cooperation Council: dominance of OXA-48 and NDM producers. Antimicrob Agents Chemother. 2014, 58:3085-3090. 10.1128/AAC.02050-13

8. Zaman Tu, Alrodayyan M, Albladi M, Siddique MI, Aljohani S, Balkhy HH: Clonal diversity and genetic profiling of antibiotic resistance among multidrug/carbapenem-resistant Klebsiella pneumoniae isolates from a tertiary care hospital in Saudi Arabia. BMC Infectious Diseases. 2018, 18:205. 10.1186/s12879-0183114-9

9. Diancourt L, Passet V, Verhoef J, Grimont PAD, Brisse S: Multilocus sequence typing of Klebsiella pneumoniae nosocomial isolates. J Clin Microbiol. 2005, 43:4178-4182. 10.1128/JCM.43.8.4178-4182.2005

10. Tamura K, Dudley J, Nei M, Kumar S: MEGA4: Molecular Evolutionary Genetics Analysis (MEGA) software version 4.0. Mol Biol Evol. 2007, 24:1596-1599. 10.1093/molbev/msm092

11. Shibl A, Al-Agamy M, Memish Z, Senok A, Khader SA, Assiri A: The emergence of OXA-48- and NDM-1positive Klebsiella pneumoniae in Riyadh, Saudi Arabia. Int J Infect Dis. 2013, 17:1130-1133. 10.1016/j.ijid.2013.06.016

12. Potter RF, Lainhart W, Twentyman J, et al.: Population structure, antibiotic resistance, and uropathogenicity of Klebsiella variicola. MBio. 2018, 9:e02481-18. 10.1128/mBio.02481-18

13. Ferreira RL, da Silva BCM, Rezende GS, et al.: High prevalence of multidrug-resistant Klebsiella pneumoniae harboring several virulence and beta-lactamase encoding genes in a Brazilian intensive care unit. Front Microbiol. 2018, 9:3198. 10.3389/fmicb.2018.03198

14. Al-Agamy MH, Shibl AM, Tawfik AF: Prevalence and molecular characterization of extended-spectrum betalactamase-producing Klebsiella pneumoniae in Riyadh, Saudi Arabia. Ann Saudi Med. 2009, 29:253-257. 10.4103/0256-4947.55306

15. Jamal WY, Albert MJ, Khodakhast F, Poirel L, Rotimi VO: Emergence of new sequence type OXA-48 carbapenemase-producing Enterobacteriaceae in Kuwait. Microb Drug Resist. 2015, 21:329-334. 10.1089/mdr.2014.0123

16. Nordmann P, Boulanger AE, Poirel L: NDM-4 metallo-beta-lactamase with increased carbapenemase activity from Escherichia coli. Antimicrob Agents Chemother. 2012, 56:2184-2186. 10.1128/AAC.05961-11

17. Quinones D, Valverde A, Rodriguez-Banos M, et al.: High clonal diversity in a non-outbreak situation of clinical ESBL-producing Klebsiella pneumoniae isolates in the first national surveillance program in Cuba. Microb Drug Resist. 2014, 20:45-51. 10.1089/mdr.2013.0021

18. Yang J, Ye L, Guo L, et al.: A nosocomial outbreak of KPC-2-producing Klebsiella pneumoniae in a Chinese hospital: dissemination of ST11 and emergence of ST37, ST392 and ST395. Clin Microbiol Infect. 2013, 19:509-515. 10.1111/1469-0691.12275

19. Uz Zaman T, Albladi M, Siddique MI, et al.: Insertion element mediated mgrB disruption and presence of ISKpn28 in colistin-resistant Klebsiella pneumoniae isolates from Saudi Arabia. Infect Drug Resist. 2018, 11:1183-1187. 10.2147/IDR.S161146

20. Ewers C, Stamm I, Pfeifer Y, et al.: Clonal spread of highly successful ST15-CTX-M-15 Klebsiella pneumoniae in companion animals and horses. J Antimicrob Chemother. 2014, 69:2676-2680. 10.1093/jac/dku217 